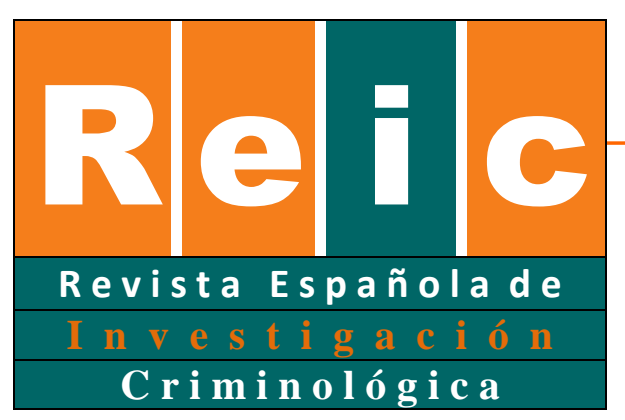

\title{
Análisis discursivo de los agentes sociales implicados en la violencia de género
}

Artículo recibido el 13 marzo 2013/Publicado el 27 mayo 2013

\author{
Lorea Arenas, Ana Isabel Cerezo \& María José Benítez ${ }^{1}$ \\ Universidad de Málaga
}

\begin{abstract}
RESUMEN
La aproximación a la metodología cualitativa a través del análisis discursivo permite a la investigación criminológica abordar realidades sociales complejas mediante la interpretación de percepciones vertidas por sujetos relacionados con una temática concreta. Nuestra investigación tiene como objetivo analizar la Ley orgánica 1/2004, de 28 de diciembre, de medidas de protección integral contra la violencia de género, a través del estudio de las argumentaciones aportadas por los agentes sociales implicados en el fenómeno. A tal fin, se han constituido diversos grupos de discusión que representan a determinados colectivos sociales, a quienes se les ha cuestionado acerca de diversos aspectos relacionados con la eficiencia, la eficacia y la efectividad de la ley. Los resultados compendian el análisis del contenido de los textos sociales obtenidos de las conversaciones y las interpretaciones integrales otorgadas a los mismos.
\end{abstract}

Palabras clave: análisis discursivo, metodología cualitativa, grupos de discusión, texto social, violencia de género.

\begin{abstract}
Approaching qualitative analysis through discourse analysis allows criminological research to deal with complex social realities by means of the interpretation of the perceptions of those involved with a specific theme. Our research intends to analyse the Law against gender violence, in force in Spain since 2005, by studying the discourses of the social agents involved in this process. To this end, several discussion groups have

\footnotetext{
${ }^{1}$ La correspondencia debe enviarse a Lorea Arenas, Instituto Andaluz Interuniversitario de Criminología, Edificio Institutos de Investigación, Campus de Teatinos, Universidad de Málaga, 29071, Málaga (Spain). Lorea@uma.es
} 
been organised, each representing a specific social group, in order to discuss their perceptions on this Law. The questions, focused on a set of predefined hypotheses and objectives, were related to the analyses of the efficiency, efficacy and effectiveness of the Law. The results, thus, synthesise and combine the different social discourses produced in each group and their interpretations from a global perspective.

Key words: discourse analysis, qualitative methodology, discussion groups, social discourse, gender violence.

\section{Introducción}

El estudio que aquí se presenta se enmarca dentro de un proyecto de investigación más ambicioso cuyo objetivo es evaluar la relación costes/beneficios del actual modelo político-criminal en materia de violencia de género, más concretamente, la eficacia, la efectividad y la eficiencia de las reformas legislativas penales y procesales aprobadas durante la última década. Al medir la eficacia nos cuestionamos, en primer lugar, si se han obtenido los objetivos de tutela perseguidos, es decir, si las últimas reformas legislativas en materia de violencia de género han alcanzado el objetivo pretendido de reducción de la violencia sobre la mujer-pareja. En segundo lugar, pretendemos analizar si la norma ha sido efectiva. La efectividad de la norma nos indica si el mandato o prohibición están siendo cumplidos, o si se ha podido reaccionar de forma adecuada al incumplimiento de la norma mediante la aplicación coactiva de la ley. Para conocer la efectividad de estas reformas se hace imprescindible conocer cuáles han sido y cómo se han activado y suministrado los recursos de intervención penal y los recursos sociales vinculados a los penales. Estos dos indicadores nos llevan en tercer lugar a plantear el análisis de la eficiencia de las reformas legales en materia de violencia de género. Queremos conocer cuáles han sido los costes ligados al objetivo principal de reducir la violencia sobre la mujer pareja.

El análisis de estos tres indicadores pragmáticos nos confirmará si la regulación jurídico-penal está logrando los efectos pretendidos. A tal fin se han recabado muy diferentes datos cuantitativos de distintos organismos. No obstante, con la pretensión de complementar nuestro estudio con otras técnicas de investigación que nos permitieran esclarecer determinados aspectos de la realidad -no contemplados en datos estadísticos-, 
decidimos aproximarnos a la metodología cualitativa a través de la técnica de investigación de los grupos de discusión y del análisis discursivo. Se trata de técnicas frecuentemente utilizadas en la evaluación de las políticas públicas ${ }^{2}$.

\section{Marco teórico}

A través de un análisis deductivo se pretende corroborar una serie de hipótesis que aluden a los tres objetivos principales del proyecto de investigación en vigor (eficacia, efectividad, eficiencia) el cual nos sirve como marco teórico de este estudio. Como se ha señalado anteriormente, la eficacia valora si se han logrado los objetivos perseguidos por la Ley, la efectividad mide el grado en que la Ley ha sido efectivamente aplicada y en qué condiciones, en definitiva, a la óptima utilización de los recursos disponibles (Osés, 2008) ${ }^{3}$ y la eficiencia se refiere a los posibles costes producidos por la apuesta de este determinado modelo político criminal.

Todas las hipótesis previstas, que se indican a continuación, van a intentar ser comprobadas desde las percepciones de los agentes, donde tienen cabida subjetividades y opiniones construidas desde determinadas posiciones sociales.

$1^{\mathrm{a}}$ - Que los agentes sociales objeto de estudio consideran que la Ley es eficaz ya que habría reducido la violencia sobre la mujer pareja.

$2^{\mathrm{a}}$ - Que los agentes sociales objeto de estudio creen que la Ley es efectiva puesto que los recursos penales y los sociales previstos en la misma están funcionando adecuadamente.

$3^{\text {a }}$ - Que los agentes sociales objeto de estudio sostienen que la Ley no es eficiente dado que:

\footnotetext{
${ }^{2}$ Nos referimos a los estudios desarrollados por el Centro de Investigaciones Sociológicas -baremos de opinión-, los grupos de discusión realizados a los usuarios o beneficiarios de un servicio y las encuestas de victimización.

${ }^{3}$ El autor propone una Evaluación Legislativa consistente en la verificación del grado de cumplimiento de los fines previstos en una ley (o política pública) y análisis de los efectos producidos. Considera que los criterios de evaluación serían dos: por un lado la efectividad jurídica y por otro los económicos-empíricos que englobarían la eficacia y la eficiencia. Nuestra división, aunque es tripartita, se aprecia asimilable, pues corresponde a cada elemento un objetivo.
} 
a. existe un progresivo rechazo de la mujer pareja a acudir al sistema penal para resolver el problema de violencia de su pareja.

b. se está produciendo una criminalización de la mujer que acude al sistema penal desinformada acerca de lo que implica poner en funcionamiento el sistema.

c. la intervención penal no está siendo igualitaria para todos, de forma que está recayendo principalmente sobre aquellos agresores con determinadas características sociales, ligadas a los grupos sociales más excluidos.

d. la denuncia del maltrato y las actuaciones de protección sobre la mujer denunciante pueden exacerbar y aumentar la agresividad sobre la mujer pareja.

e. se estaría produciendo una sobrecarga de las capacidades de gestión de las órdenes y penas de alejamiento y una saturación de los juzgados penales por la ejecución de penas que siempre llevan emparejadas una orden de alejamiento.

\section{Método}

El diseño del estudio está compuesto por tres fases sucesivas:

En la primera fase hemos constituido cuatro grupos de discusión (Gil, 1993) correspondientes a cuatro de los agentes sociales de interés en nuestro trabajo: asociaciones de víctimas, sujetos que sustituyen una pena privativa de libertad por haber cometido un delito en materia de violencia de género, operadores jurídicos y Fuerzas y Cuerpos de Seguridad.

En el grupo de operadores jurídicos la muestra ha quedado conformada por tres abogados de familia, dos magistrados pertenecientes a Juzgados de violencia sobre la mujer, dos expertos en asistencia jurídica y psicológica a víctimas y una fiscal de violencia de género.

En operadores policiales se ha contado con la participación de dos policías locales, tres policías nacionales y dos guardias civiles de la ciudad de Málaga y su provincia. 
El tejido asociativo estuvo representado por una asociación de ayuda a menores víctimas indirectas de la violencia de género, por una representante de la Secretaria de Igualdad de Género de la Diputación de Málaga, por dos asociaciones pro-igualdad que, además, luchan contra la violencia hacia la mujer y por una fundación de ayuda a mujeres maltratadas.

Respecto a los victimarios, el grupo quedó constituido por catorce personas que se encontraban realizando un curso en materia de violencia de género en el Centro de Inserción Social de Málaga. Los criterios adoptados en el diseño de cada grupo han sido establecer un nivel básico de homogeneidad intragrupal y seleccionar un número determinado de participantes que no se conociesen entre sí (Mena y Méndez; 2009, Ibáñez, 2000).

El valor del método -en relación a nuestros objetivos de investigación- reside esencialmente en la construcción de un discurso colectivo de un perfil social determinado a partir de la dinámica de la discusión grupal en un contexto cuasi natural; esto es, la discusión grupal es el ámbito natural cotidiano en que las opiniones se generan, intercambian y expresan. Siguiendo esta lógica, los grupos de discusión sirven tanto para estudiar las opiniones individuales como para analizar las de un colectivo; en nuestro caso, el objetivo es el segundo, esto es, comprender las percepciones de los colectivos mencionados (Flick, 2007). Así mismo, la muestra es de tipo intencional y los discursos resultantes son provocados ${ }^{4}$. Las reuniones se han llevado a cabo en lapsos temporales diferentes y en espacios de interacción orientados a fomentar que los distintos grupos se expresasen libremente. La dinámica mantenida en todos los casos ha sido activa y participativa, dado que el tema escogido ha tenido la suficiente capacidad de despertar interés y generar un alto grado de implicación entre los grupos.

Aun así, en cada dinámica se aprecian una serie de matices propios, fruto de la posición social que ostentan los grupos ${ }^{5}$.

\footnotetext{
${ }^{4}$ Se trata de discursos no naturales generados en el marco de una investigación.

${ }^{5}$ Tras el intento fallido de formar un quinto grupo de discusión con víctimas de violencia de género por no obtener el mínimo necesario para constituirlo y habiendo realizado tres entrevistas personales a víctimas, se ha tomado la decisión de incluir éstas en el material de análisis de nuestro estudio. Las tres entrevistas no han sido consideradas tampoco como un grupo triangular. Éste ocuparía una posición central entre las entrevistas personales y los grupos de discusión (Ruiz, 2012). El menor número de participantes del grupo triangular condiciona la dinámica y, en este sentido, "hace que no se erija en la representación simbólica del lugar de pertenencia social de los asistentes” (Roldán, 1998, 137-138).
} 
En la segunda fase hemos procedido a llevar a cabo el análisis del contenido y la interpretación sociológica del discurso ${ }^{6}$. La metodología empleada en el análisis del contenido está basada en una fragmentación de los corpus del texto extraídos de las transcripciones resultantes de las conversaciones. La información obtenida de la muestra ha sido analizada atendiendo a distintos criterios metodológicos. Con relación al análisis del contenido, se ha llevado a cabo una aproximación analítica que descompone y fragmenta el corpus del texto en "unidades elementales de análisis". Los corpus del texto correspondientes a grupos de discusión han sido fragmentados y codificados utilizando una herramienta de análisis cualitativa textual denominada ATLAS.ti (The Qualitative Data Analysis \& Research Software) ${ }^{7}$.

En el proceso de transcripción se ha incorporado la información dinámica de los sociogramas en los que se captaron las principales posiciones discursivas, las diferencias u oposiciones entre los grupos o sus segmentos, así como toda la información no verbal. El tratamiento de los datos se ha realizado codificando la información escrita de los textos. Para ello, se ha enumerado cada frase del texto y se ha asociado un código a las diferentes citas susceptibles de ser agrupadas bajo un mismo concepto. Nos referimos a un trabajo de síntesis y de abstracción (Conde, 2009) donde se ha codificado y categorizado unos textos base para integrarlos en categorías o familias que se configuran como un nivel superior del análisis conceptual y, que a su vez, ha posibilitado su agrupación en torno a unas temáticas generales.

En relación al análisis interpretativo, éste ha sido abordado desde una perspectiva integral sin atender a la descomposición o fragmentación de los discursos, si bien, se han considerado con carácter previo las posiciones, los roles y las prácticas discursivas de los sujetos. Hemos procedido a analizar el texto como una totalidad dotándole de un sentido e interpretación sociológica (Ricoeur, 1995), teniendo en cuenta su dimensión informativa (Ruiz, 2009), esto es, considerando que los discursos son

\footnotetext{
${ }^{6}$ El análisis pretende alcanzar un conocimiento objetivo del discurso partiendo de las intersubjetividades discursivas en un proceso de continua retroalimentación. El análisis sociológico del discurso es una técnica que pretende caracterizar el discurso y, el mismo, no se ha llevado a cabo desde una perspectiva semiótica (Hallyday, 1982), ni pragmática.

${ }^{7}$ Con respecto a las entrevistas con las víctimas, no se ha codificado su contenido al no tratarse de grupos de discusión y no se ha realizado la comparativa.
} 
producidos por sujetos que están en contacto con una realidad en particular y, por lo tanto, poseen un conocimiento de la misma (Íñiguez, 2006).

\section{Resultados}

En el presente apartado se definen en primer lugar todas las categorías temáticas resultantes del análisis del contenido. En segundo lugar, se desgrana de forma descriptiva el contenido de cada una de ellas para determinar su presencia o ausencia en los discursos de los grupos. Por último, se procede a realizar una aproximación interpretativa del análisis del contenido.

\subsection{Categorías temáticas}

Del análisis de los textos base se ha obtenido un total de 80 códigos distribuidos en diversas categorías agrupadas en torno a diferentes temas. Cada categoría está compuesta por un determinado número de códigos pudiéndose incluir éstos en similares o distintas categorías temáticas. La frecuencia del código -el número de veces que se repite-, pone de relieve que una determinada idea ha sido mencionada en escasas o múltiples ocasiones. A continuación se muestran las 16 temáticas establecidas e integradas por una o dos categorías, así como por sus respectivos códigos. Anticipamos, que los temas no han emergido en todos grupos ni se han mencionado con la misma frecuencia.

Las categorías temáticas abordadas en el presente estudio son:

a. La concepción de la violencia de género. Inserta aquellas partes del corpus que conciben a la violencia de género como un problema estructural de la sociedad, o bien, como un conflicto puntual de pareja.

b. La justificación de la situación. Alude a la creación y el mantenimiento de la situación de violencia de género, esto es, recoge los razonamientos que los sujetos aportan para explicar los actos y las causas de la violencia. 
c. La fundamentación de la Ley. Compendia las opiniones sobre los motivos o la filosofía inspiradora de la norma. Engloba dos categorías dicotómicas: la fundamentación de la ley es correcta y la fundamentación de la ley es errónea.

d. El desarrollo parcial de la Ley. Indica que la norma no se ha desarrollado en su totalidad.

e. El automatismo de los procesos. Señala que los procesos policiales y judiciales son concebidos como mecanizados y/o automáticos. Dos son las categorías extraídas: el automatismo policial es negativo y el automatismo judicial es negativo.

f. La descoordinación de la Ley. Reseña que los operadores no están bien organizados y no comparten información relevante de su práctica diaria. No hay categorías desglosadas en sentido positivo.

g. El trato policial. Se refiere al trato otorgado por los agentes en los procesos de denuncia. Engloba dos categorías contrapuestas: el trato policial es correcto y el trato de policial es incorrecto.

h. La interposición de la denuncia. Aborda la denuncia desde una doble perspectiva: razones a favor de la denuncia y razones en contra de la denuncia.

i. La orden de alejamiento. Trata una serie de aspectos vinculados a la medida cautelar y ha dado lugar al establecimiento de una única categoría: razones en contra de la orden de alejamiento.

j. Los recursos previstos en la Ley. Alude a que una amplia mayoría de las medidas previstas en la norma no han sido puestas en marcha o están funcionando parcialmente. Obtenemos dos categorías, a saber: los recursos previstos en la Ley son adecuados y los recursos previstos en la Ley son inadecuados. 
k. El colapso del sistema penal. Reseña que se ha asumido una carga de trabajo desproporcionada a los medios previstos en la norma.

1. Los grupos de presión. Encauza las opiniones que mencionan al poder ideológico y político como causante de los problemas o ventajas de la norma. Se distinguen dos categorías: los grupos de presión perjudican a la Ley y los grupos de presión favorecen a la Ley.

m. La mediación. Recoge las opiniones acerca de la conveniencia de aplicar la mediación como forma de justicia restaurativa en casos de violencia de género.

n. Los programas para agresores. Engloba las opiniones acerca de la eficiencia de los programas socioeducativos.

ñ. Los efectos negativos en el ámbito familiar. Señala que la norma ha ocasionado efectos negativos en el núcleo familiar de victimarios y víctimas.

o. Efectos criminógenos. Indica que las medidas previstas en la Ley pueden aumentar la agresividad sobre la mujer pareja y, en ocasiones, generar nuevos casos de violencia.

\subsection{Análisis del contenido del grupo de las Fuerzas y Cuerpos de Seguridad}

Según se muestra en la figura 1, las categorías temáticas más relevantes de este grupo son: el automatismo de los procesos (51 códigos), los grupos de presión (37 códigos), el desarrollo incompleto de la Ley (32 códigos), la descoordinación de la Ley (20 códigos) y el concepto de violencia de género (20 códigos). 
Figura $n^{0}$ 1. Representación de las categorías temáticas del grupo de discusión de Fuerzas y Cuerpos de Seguridad.

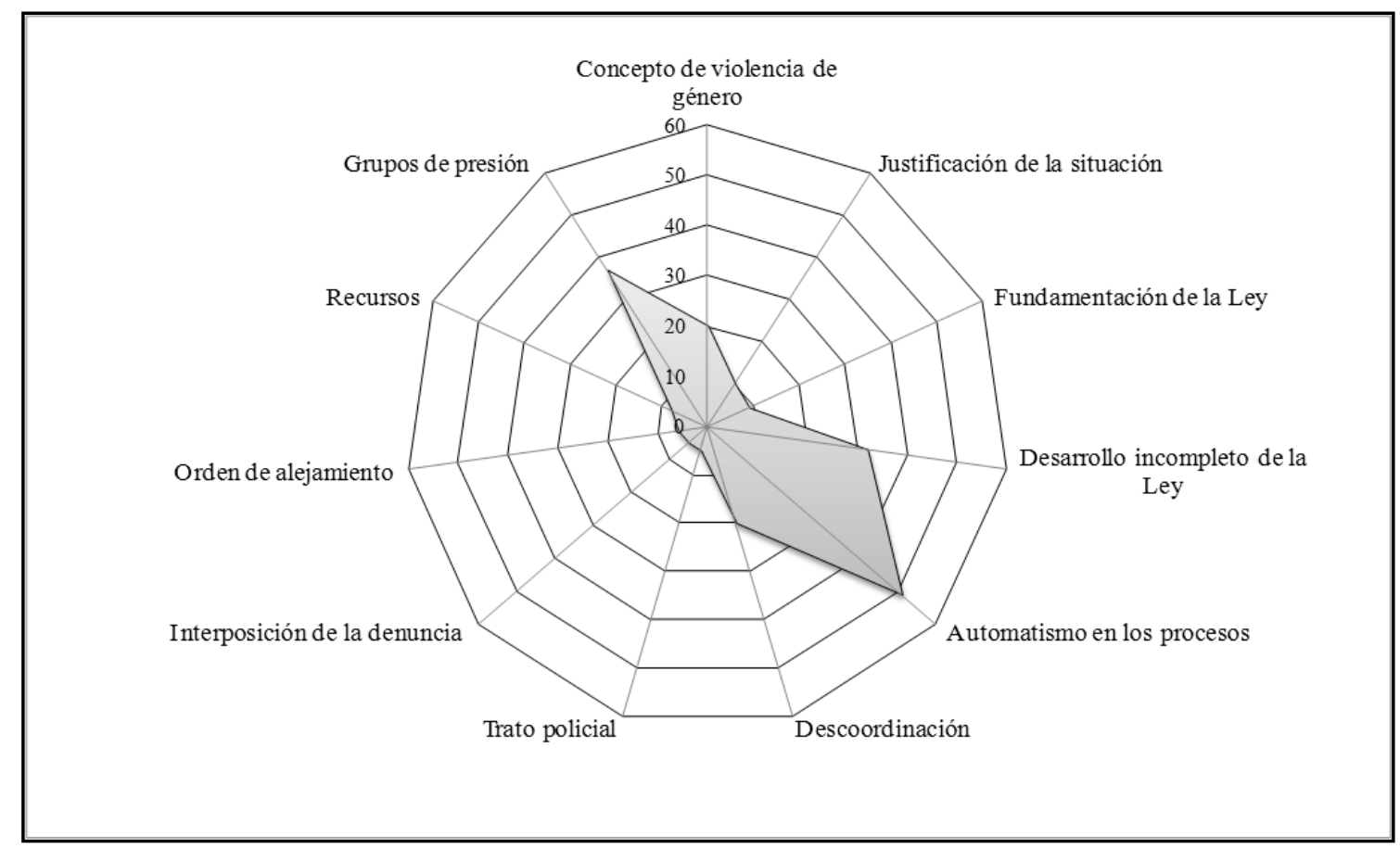

En primer lugar, los operadores policiales reconocen que sus actuaciones están marcadas por el automatismo. Destaca la connotación negativa de las valoraciones vertidas en torno a la celeridad y a la superficialidad de los procesos. A modo de ejemplo, se hace referencia a la valoración policial del riesgo. Ésta se realiza con información procedente de la víctima y sin apenas datos, hecho que le otorga un cierto carácter discrecional. Igualmente, se asocia el automatismo a la reticencia de la víctima a denunciar, puesto que a ésta le asaltan dudas al desconocer el proceso de denuncia, debido a su celeridad.

En segundo lugar, los grupos de presión influyen en la toma de decisiones de la política policial. Los operadores policiales muestran cierto temor a la alarma social ocasionada por un nuevo caso de muerte, hecho que favorece que se actúe de forma casi automática y a que se inste a la mujer a denunciar para evitar riesgos. Se refieren a este hecho como la política del riesgo cero. Muestra de ello es la instauración de la política: la denuncia es necesaria siempre, circunstancia que fomenta el automatismo en el proceso y va en detrimento de la investigación de los casos. Por último, se afirma que es necesario investigar los casos en profundidad con la ayuda de equipos especializados, 
así como instruir a los agentes en violencia de género. Medidas que, de adoptarse, evitarían la mecanización del proceso.

La tercera categoría más mencionada por el grupo ha sido el desarrollo incompleto de la ley. Se alude a que los medios son insuficientes para su aplicación práctica y a que no hay unidades policiales específicas para atender los casos de violencia de género. En concreto, consideran que queda por desarrollar el ámbito de la educación y de la formación especializada para profesionales previstos en la ley. A su vez, el escaso desarrollo de la norma está ligado a una falta de retroalimentación de la información sobre los casos de violencia que tratan los operadores policiales y judiciales. Realidad que queda reflejada en la temática descoordinación.

Por último, el concepto de violencia de género es entendido en torno a dos concepciones distintas. Por un lado, se entiende la violencia como un fenómeno estructural (16 códigos) y, por otro, como un conflicto puntual de pareja (4 códigos). La visión mayoritaria sostiene que la violencia de género no es un delito pasional y puntual, sino que está favorecida por un conjunto de roles y estereotipos transmitidos a través de una socialización desigual. La violencia de género entre adolescentes vendría a constatar el carácter estructural de la misma y su perpetuidad en el tiempo.

Es relevante mencionar que este grupo no dio importancia a las siguientes temáticas: la justificación de la situación (10 códigos), la fundamentación de la Ley (9 códigos), los recursos (8 códigos), la orden de alejamiento (6 códigos), el trato policial (5 códigos) y la interposición de la denuncia (5 códigos).

En cuanto a la justificación de la situación, se indica que las situaciones personales que motivan la aparición de la violencia de género están marcadas por un alto nivel de emocionalidad $\mathrm{y}$, en este sentido, se hace complicado distinguir las situaciones de violencia de las que no lo son. Respecto a la fundamentación de la Ley, se advierten opiniones divididas que señalan sus aspectos positivos (3 códigos) y negativos (6 códigos). En relación a los recursos, éstos son definidos como inadecuados ${ }^{8}$. Consideran que no funcionan porque son insuficientes para poner en marcha el conjunto de medidas previstas en la Ley. Prueba de lo anterior es que los agresores violan las órdenes de alejamiento. Además, entienden que la descoordinación

\footnotetext{
${ }^{8}$ En relación a esta categoría existen razones que indican su inconveniencia (6 códigos) y su correcto funcionamiento ( 2 códigos).
} 
entre los distintos operadores es debida a una falta de medios y a la ausencia de un escalón intermedio entre la actuación policial y judicial. De la misma manera, aprecian que los recursos están distribuidos de forma inadecuada. En cuanto a la orden de alejamiento, se han aportado razones negativas de su operatividad. En concreto, se pone de relieve que los agresores violan la orden de alejamiento y que existe una falta de seguimiento de su cumplimiento. Con relación al trato policial, el tema se presenta vinculado al código: es necesario investigar los casos. Este código es referido por los policías como un automatismo en el sentido de que no conocen si los hechos que motivan la detención del sujeto puede tener una base real o, si por el contrario, están cometiendo una injusticia. La percepción de las correctas actuaciones de su trabajo viene dada a posteriori, cuando han tenido constancia de la realidad de la entidad de los hechos. Respecto a la interposición de la denuncia, todas las razones aportadas se muestran a favor de ésta.

\subsection{Análisis del contenido del grupo del tejido asociativo}

En la figura inferior, se contemplan las temáticas emergentes del grupo de asociaciones, siendo el desarrollo incompleto de la Ley (36 códigos), los recursos (34 códigos), el concepto sobre la violencia de género (17 códigos) y la fundamentación de la Ley (17 códigos) las más referidas. 


\section{Figura n². Representación de las categorías temáticas del grupo de discusión del} tejido asociativo.

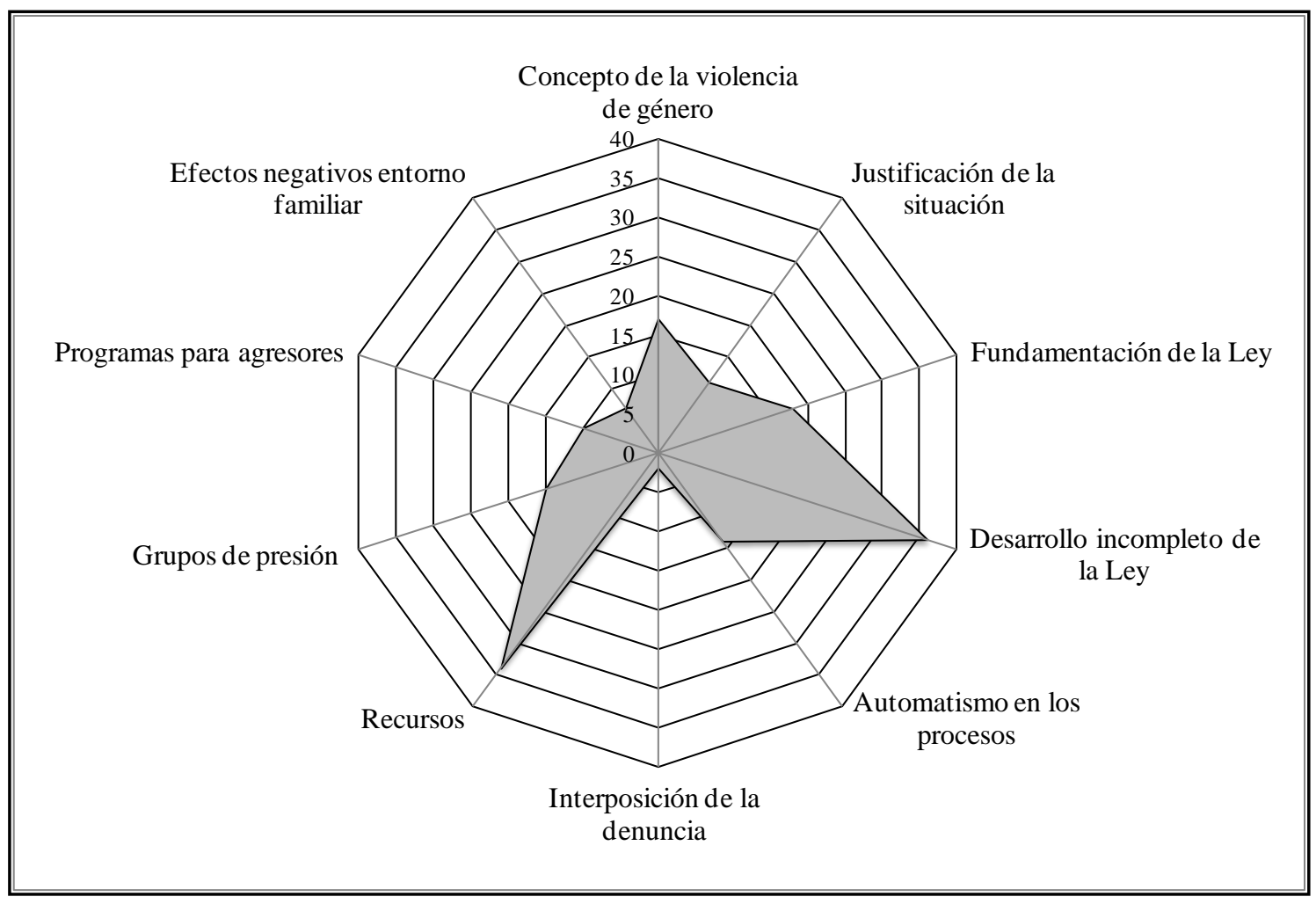

El tema desarrollo incompleto de la Ley indica que las medidas previstas en la norma no se han desarrollado en su totalidad. La educación no ha sido suficientemente desarrollada, muestra de ello es la existente violencia sexista entre adolescentes.

En segundo lugar, se menciona el escaso desarrollo de los recursos. Es destacable que sólo se señalan dos tipos de recursos que funcionan correctamente, a saber: los recursos en el ámbito del empleo y ayudas, y los recursos en el ámbito de la urgencia sanitaria. Por el contrario, se apunta que no se han destinado recursos al ámbito del menor víctima indirecta y al plan de viviendas. Así mismo, se indica que no está desarrollada la formación específica para profesionales y que no hay medios suficientes para su aplicación práctica. Por último, se hace alusión a la pulsera de control telemático junto a la medida cautelar de alejamiento como un recurso sin desarrollar, indicándose que se han adquirido más pulseras de las que están siendo utilizadas. En definitiva, se pone de manifiesto que el planteamiento de la Ley es acertado, sin embargo, no se puede valorar adecuadamente en un plano práctico por la falta de recursos. 
En tercer lugar, se mencionan el concepto de violencia de género y la fundamentación de la Ley. Con respecto al primero, el tejido asociativo comparte una visión unánime del mismo. Lo definen como un problema estructural y no como un conflicto puntual. De ahí los casos de violencia de género entre adolescentes y entre las parejas de la tercera edad, lo cual es indicativo de una transmisión de roles y estereotipos sexistas perpetuados a lo largo del tiempo. En cuanto a la fundamentación de la norma, se afirma que la misma está bien fundamentada, con una excepción, posee un cierto carácter paternalista. En sentido positivo, las muertes actuales no pueden interpretarse como un indicador de ineficacia. Consideran que la Ley posee una orientación preventiva general cuyos frutos serán visibles con el paso del tiempo. A su vez, opinan que la Ley sensibiliza y saca a la luz pública un problema que ha estado relegado al ámbito de lo privado, en consecuencia, la ley consigue concienciar a la sociedad y dar una mayor visibilidad al problema. Además, la norma es positiva porque protege a la mujer y ello es debido a su carácter integral y unificador. Todo este conjunto de razones positivas hace que la norma sea útil y acorde a los fines por los cuales ha sido creada.

Hay una serie de temáticas que han sido poco referenciadas, a saber: el automatismo (14 códigos), la justificación de la situación (11 códigos), los programas de agresores (10 códigos), los grupos de presión (8 códigos), los efectos negativos en el ámbito familiar (7 códigos) y la interposición de la denuncia (2 códigos). El automatismo pone de relieve que la mujer es victimizada durante el proceso jurídicopenal, indicándose la existencia de victimización secundaria. Así mismo, se reseña que los operadores que tratan con la mujer durante todo el proceso no disponen de formación especializada suficiente. La justificación de la situación de violencia es abordada desde la perspectiva teórica de la socialización diferencial (Espinar, 2007) ${ }^{9}$. La categoría de los programas de agresores es mencionada únicamente por este grupo, el cual debate acerca de su eficacia. Respecto a los grupos de presión, el tejido asociativo posee una opinión unánime. Se pone de relieve que ciertas ideologías

\footnotetext{
${ }^{9}$ El imaginario colectivo está construido sobre una serie de creencias, normas y estereotipos de género desarrollados en un contexto social determinado. En este sentido, la masculinidad y la feminidad son productos sociales que guardan unas marcadas diferencias y una relación jerárquica entre géneros. La educación transmitida por las agencias de socialización formales e informales vendrían a transmitir y perpetuar éstas, favoreciendo en determinadas situaciones la aparición de situaciones de violencia sobre la mujer por el simple hecho de serlo.
} 
políticas y medios de comunicación social están perjudicando a la actual Ley. En último lugar se mencionan los efectos negativos en el ámbito familiar -se alude a la victimización de menores en el ámbito familiar y al escaso desarrollado que la norma en ese ámbito- y la interposición de la denuncia -siendo todas las razones aportadas favorables a la misma-.

\subsection{Análisis del contenido del grupo de victimarios}

Los agresores han tratado un número menor de temas en comparación al resto de grupos. Destaca la categoría del automatismo en los procesos (49 códigos), seguida de la fundamentación de la Ley (19 códigos) y de la justificación de la situación (18 códigos).

\section{Figura n³. Representación de las categorías temáticas del grupo de discusión de victimarios}

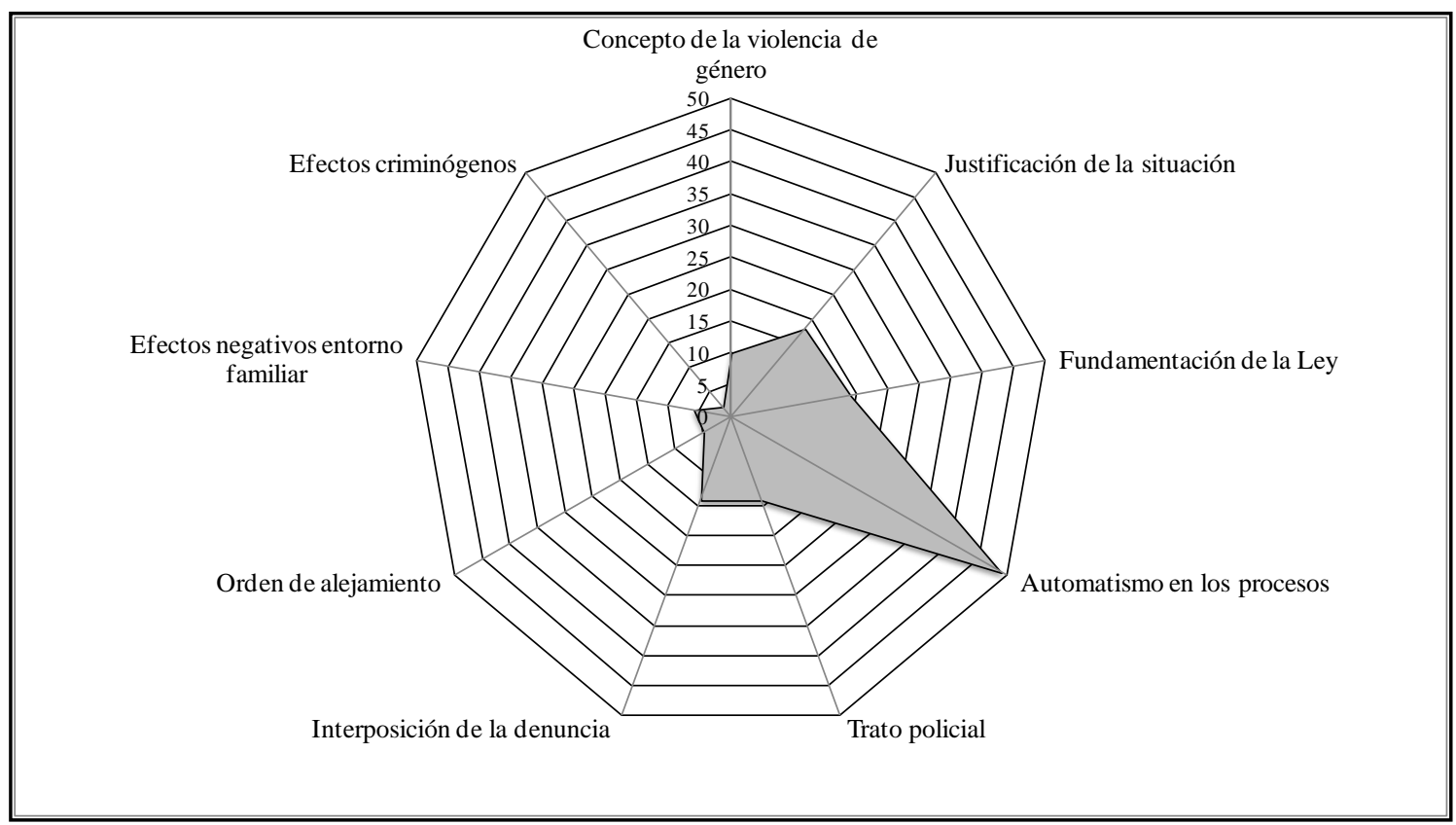

El automatismo en los procesos señala que el proceso judicial es experimentado con mucha celeridad y con una deficiente atención letrada. Los agresores opinan que es necesario investigar los casos y distinguir aquellos propios de violencia de género de los 
que no lo son. Indican que la mujer no conoce las consecuencias de la interposición de la denuncia y que ellos no tienen claro los motivos de la misma. Contemplan a la mujer y el acto de denuncia como una situación de obligatoriedad en la que los trabajadores sociales y los operadores judiciales instan a la mujer a denunciar.

En segundo lugar, los victimarios vierten opiniones muy negativas de la fundamentación de la ley. Apuntan que es discriminatoria para los hombres, injusta con los más débiles e inapropiada. De igual manera, señalan que es necesario distinguir a las falsas de las verdaderas víctimas puesto que la Ley no está diseñada para distinguir a los hombres agresores de los que no lo son. Ésta se limita a considerar a todo hombre agresor por razón de su sexo.

En tercer lugar, el grupo de agresores se aproxima a la Ley de forma egocéntrica haciendo numerosas alusiones a las historias personales y familiares que justifican su situación. Los razonamientos que aportan los agresores para explicar los actos de violencia y sus causas están impregnados de continuas excusas o técnicas neutralizadoras. Mencionan que las dificultades familiares actúan como factores de riesgo, justificando su comportamiento en los problemas económicos y de pareja. Tienden a minimizar los hechos acontecidos y a eximir su responsabilidad. Justifican las situaciones de violencia como sucesos fortuitos o sin entidad suficiente como para provocar la intervención policial y judicial. Culpabilizan a sus parejas de la situación experimentada, así como puntualizan que ellas subsisten gracias a la manutención de ellos. Refieren que han perdonado a sus parejas por haberles denunciado y que sus casos no pueden ser denominados de violencia de género.

A continuación, hallamos tres temáticas relevantes insertas en un plano secundario respecto de las anteriores: la interposición de la denuncia (14 códigos), el trato policial (14 códigos) y el concepto de violencia de género (10 códigos). En relación a la primera, todas las razones que se expresan son negativas, esto es: la víctima se ve obligada a denunciar, desconoce las consecuencias de la denuncia e interpone denuncias falsas. En cuanto al trato policial, se define como incorrecto y, puntualmente, se hace referencia al maltrato policial xenófobo. En contraposición, se señala el trato de favor recibido por la policía en el caso de que el detenido sea un familiar o conocido. Respecto al concepto de violencia de género, éste es definido como un conflicto puntual de pareja. 
En último lugar, se mencionan los efectos negativos en el ámbito familiar (6 códigos) -indican un deterioro o pérdida de la relación con los hijos-, la orden de alejamiento (5 códigos) -afirman haber convivido con sus parejas durante el tiempo que la medida o pena estaba vigente- y los efectos criminógenos (2 códigos) -indican que asociar la privación de la patria potestad a la condena puede generarlos-. Resaltan como temáticas ausentes: la fundamentación de la Ley, los recursos, los grupos de presión y el colapso del sistema judicial.

\subsection{Análisis del contenido del grupo de operadores jurídicos}

Tal y como se contempla en la figura $\mathrm{n}^{\circ} 4$, los operadores jurídicos han tratado una amplia variedad temática, siendo especialmente citadas la fundamentación de la Ley (38 códigos), el desarrollo incompleto de la Ley (30 códigos) y los recursos (30 códigos). A su vez, emergen dos nuevos temas característicos de este grupo: el colapso del sistema judicial (21 códigos) y la mediación (5 códigos). 
Figura n4. Representación de las categorías temáticas del grupo de discusión de los operadores jurídicos.

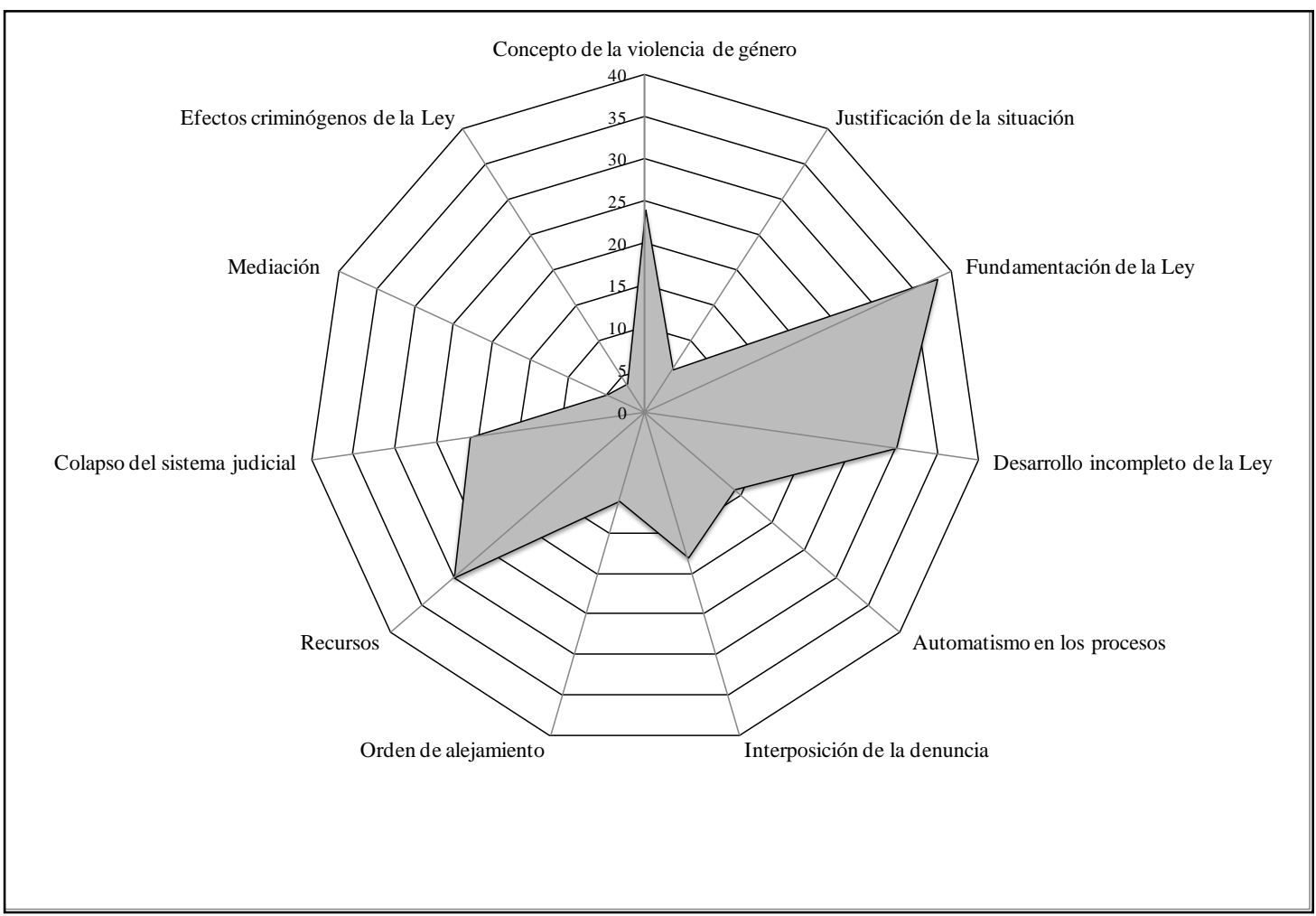

Con respecto al fundamento de la Ley encontramos divergencias de opiniones, a saber: fundamento erróneo de la Ley (31 códigos) y fundamento acertado de la Ley (7 códigos). Se citan los siguientes aspectos negativos de la norma: la Ley es de difícil interpretación, la Ley es discriminatoria para los hombres, la Ley no es eficaz porque en la actualidad existen casos de violencia de género, la Ley es inapropiada, es necesario distinguir a las falsas de las verdaderas víctimas y se está abordando un problema social con una ley penal. En sentido positivo se apunta: las muertes no son un indicador de ineficacia, la Ley será eficaz con las nuevas generaciones, la Ley es positiva porque es unificadora e integral, la Ley es positiva porque protege a la mujer y, por último, la Ley sensibiliza y saca a la luz pública el problema de la violencia de género.

Según este grupo, la Ley recoge una serie de elementos de tipo social, económico, laboral, en el ámbito sanitario, entre otros, que no están siendo desarrollados de forma adecuada debido en parte a una falta de recursos. La aplicación 
de la ley en el ámbito educativo es anecdótica quedando un largo camino por recorrer. Con respecto a ello, la violencia de género entre adolescentes es un síntoma de la falta de desarrollo de la parte educacional contemplada en la Ley en los artículos 9 y ss. De igual forma, se señala que no se ha desarrollado el ámbito de la formación en género dirigida a profesionales, así como que no se han creado unidades especializadas. En definitiva, estas medidas se han dejado de lado y se ha empezado por la parte penal.

Los recursos son descritos de manera unilateral como insuficientes. En concreto, se apunta que los recursos son insuficientes para su aplicación práctica, que las falsas víctimas colapsan los recursos en detrimento de las verdaderas, que es necesaria la creación de unidades especializadas y de protocolos de actuación, que no existe una formación específica para los profesionales y que los recursos están distribuidos de manera inadecuada.

A colación, el colapso en el sistema judicial es un tema muy recurrido por los operadores jurídicos. Se hace referencia al colapso experimentado por el sistema judicial con motivo de la sobrecarga funcional causada por el número de casos diarios que los operadores deben atender. Perciben que son numerosos los casos de violencia de género que están llegando al sistema y opinan que se deberían establecer mecanismos que permitiesen filtrar estos casos. En este sentido, se efectúa una distinción de aquellas víctimas sobre las que ha de recaer todo el peso del sistema penal, así como los recursos previstos en la ley y, aquellas otras, que acuden a los juzgados sin un problema de entidad suficiente como para necesitar de intervención judicial. El filtro evitaría la sobrecarga de facto de los juzgados.

Como temas emergentes situados en un nivel secundario hallamos los siguientes: el concepto de violencia de género (24 códigos), la interposición de la denuncia (18 códigos), el automatismo (14 códigos) y la orden de alejamiento (11 códigos). La violencia de género es descrita en más ocasiones como un conflicto puntual de pareja (18 códigos) que como un fenómeno estructural (6 códigos). De otra parte, la interposición de la denuncia ha sido abordada desde dos perspectivas, aportándose razones a favor de denunciar (8 códigos) y razones en contra (10 códigos). En cuanto al automatismo en el proceso, se indica que es necesario un mayor asesoramiento de otros profesionales a los medios jurídicos, puesto que en el poco tiempo que duran los procedimientos no todos ellos tienen acceso a informes específicos del caso. Por último, 
la orden de alejamiento se percibe como ineficaz y se aportan razones en contra de ésta. En particular, se menciona que la víctima se acoge a su derecho a no declarar para evitar la imposición de la orden de alejamiento y que debería existir mayor discrecionalidad en su imposición. Así mismo se afirma que los agresores la quebrantan constantemente.

En último lugar, encontramos una serie de temáticas escasamente referenciadas, tales como: la justificación de la situación (6 códigos), la mediación (5 códigos) y los efectos criminógenos (4 códigos). Se justifica el mantenimiento de la situación a causa de la situación de dependencia económica de la mujer y se señalan los efectos criminógenos que puede ocasionar la norma si ésta vincula la condena a una pérdida automática de la patria potestad. La mediación se presenta como una temática novedosa y debatida, otorgándose razones a favor ( 2 códigos) y razones en contra de la misma (3 códigos). Destaca el grupo de presión como temática ausente.

\subsection{Análisis del contenido de las entrevistas de las víctimas}

Las víctimas inciden en temáticas similares a las tratadas en los grupos. Con carácter general, valoran la Ley como positiva y eficaz: "lo bueno de esta Ley es que a pesar de que estos comportamientos pertenezcan al ámbito doméstico ahora al menos la gente lo sabe, se ha hecho público". En cuanto al concepto de violencia de género, refieren que el maltrato es fruto de un proceso educacional y reconocen comportamientos de sus parejas en los ascendientes de éstas e incluso es sus propios hijos: "lo veo en mis hijos, en mi hijo mayor, tiene cosas de él, ve el modelo del padre", "mi hijo también ha sacado cosas del padre". Las mujeres justifican la creación y mantenimiento de las situaciones de maltrato desde su propia experiencia. Autoevalúan las circunstancias vividas y no comprenden cómo han aguantado y perdonado los comportamientos de sus parejas: "te aleja de tus amigos, de tu familia, es una escalada que va poco a poco, en concordancia con las secuelas físicas y psicológicas".

En cuanto al automatismo, la asistencia letrada se configura como una pieza clave del proceso que determina el grado de victimización secundaria experimentado. El hecho de que los juicios sean rápidos no es algo problemático, puesto que la mujer es capaz de razonar y pensar. Afirman ser conscientes del proceso y la celeridad es concebida como positiva, ya que apacigua la sensación de revivir la situación. Señalan 
que durante el juicio no pudieron expresarse ni aclarar las circunstancias de su caso: "yo sentí mi juicio como un circo", "mucha impotencia", "no puedes hablar ni comentar todo lo que había pasado". En este sentido, tanto la intervención letrada, como la información facilitada por los agentes policiales durante el momento de la interposición de la denuncia, son consideradas cruciales.

El trato policial es valorado como atento, profesional y eficaz. Las víctimas se han sentido muy apoyadas por la policía y la orden de alejamiento les parece fundamental: "la orden de alejamiento genial, yo no le he vuelto a ver cara a cara". Todas las víctimas coinciden en afirmar que la Ley dificulta el acceso de los agresores a la víctima, si bien, reconocen que ningún mecanismo puede ser lo suficientemente eficiente como para detener un ataque. Los fines de disuasión efectiva de la orden quedan mermados por el conocimiento público de los casos de muerte, aunque esa percepción cambia cuando es impuesta sobre uno mismo. Por lo tanto, no se debería desestimar la orden basándose en su incapacidad práctica, teniendo en cuenta que genera una sensación subjetiva de protección.

En relación a los recursos, mencionan que las mujeres que acuden a juzgados de violencia de género obtienen menos beneficios en comparación con los juzgados de familia. El recurso que mejor funciona es la intervención psicológica a menores. Sin embargo, las víctimas que sufrieron violencia con anterioridad a la Ley, opinan que sus hijos no fueron debidamente evaluados. Es decir, parece que los recursos para niños han mejorado desde el año 2003. De otro lado, los programas para agresores son valorados como ineficaces puesto que no creen en la posibilidad del cambio conductual. En cuanto al tema de la mediación, mantienen una opinión unánime: “no, no es buena. Siempre será la ley del embudo. Una mediación nunca va a funcionar, siempre consistirá en que nos adaptemos nosotras. Poseen una agresividad que es difícil de compatibilizar con una actividad de mediación". Por último, se mencionan los efectos negativos en el ámbito familiar ocasionados por la norma. Parece que los problemas vendrían dados por la falta de cumplimiento de los progenitores del régimen de visitas a los niños. Afirman que no existen consecuencias legales si es infringido y que la Ley no ofrece una respuesta a este problema. 


\subsection{Una aproximación interpretativa al análisis del contenido.}

Cada agente ostenta una posición social que determina en gran parte la interpretación discursiva. Así, los operadores jurídicos poseen un alto nivel de conocimiento de la legislación y del sistema judicial, su repertorio es profesionalizado y expulsan las emociones del discurso. Es por ello que hayamos un mayor número de referencias que hacen alusión al colapso del sistema penal y a la necesidad de filtrar los casos en este grupo. Los operadores jurídicos perciben el colapso y la sobrecarga de trabajo, pero no los procesos psicológicos que se hallan detrás de los casos. Así mismo, tampoco poseen un conocimiento detallado de los casos que les permita discernir con claridad ante qué situaciones de violencia de género se encuentran. Por lo tanto, su conocimiento de la realidad les hace cuestionarse acerca del concepto de violencia y de su interpretación legal. En este sentido, no abordan el problema de la violencia de género desde una perspectiva de género, puesto que construyen la violencia como un conflicto puntual de pareja y no como un fenómeno que forma parte de un proceso. La lógica profesional de optimización de recursos -junto al reconocimiento implícito y explicito de la violencia de género como un problema no estructural- abre el debate acerca de las falsas y verdaderas víctimas. Esta concepción denota que existe una referencia indirecta al concepto de violencia de género como conflicto matrimonial puntual definiendo como falsas a aquellas mujeres que no provienen de una situación verdadera de maltrato y que harían un abuso indebido del sistema. Además, otorgamos una segunda interpretación al código falsa víctima, y es su carácter de reproche y culpabilización de este tipo de mujeres que saturan el sistema y restan recursos a las verdaderas o merecedoras víctimas. Esta intersubjetividad puede a su vez integrarse en una concepción social mayor compartida que cuestiona el concepto de la violencia de género y la veracidad de las situaciones de denuncia. A colación, emerge el tema de la mediación. Consideran que si la violencia de de género es un conflicto puntual de pareja éste puede ser solventado mediante el diálogo. De modo contrario, los grupos que conciben de forma exclusiva a la violencia de género como un fenómeno estructural, no mencionan la mediación o indican su inconveniencia.

Los operadores policiales valoran la eficacia, la efectividad y la eficiencia de la Ley señalando sus aspectos técnicos y ejecutorios, en concreto, el automatismo policial 
y la falta de retroalimentación de la información entre los agentes. Igualmente, construyen un concepto de violencia de género excluyendo parcialmente la perspectiva de género, puesto que se reconoce su carácter estructural en mayor grado. Además, reconocen -en mayor o menor medida- que es necesario el desarrollo de la norma en el ámbito educativo. Con ello están asumiendo que la violencia de género es un fenómeno estructural que se transmite a través de una socialización desigual entre sexos. En este orden de ideas, los discursos de los operados jurídicos y policiales están compartiendo de forma implícita una similar perspectiva de género al indicar que es necesario desarrollar el ámbito educativo.

El tejido asociativo analiza la Ley desde una perspectiva de género dando una gran relevancia al elemento ideológico, en consecuencia, comparten una visión unánime de la violencia de género como problema estructural y no valoran la posibilidad de una actividad de mediación. Analizan la eficacia, la efectividad y la eficiencia de la ley en relación al nivel de satisfacción de las víctimas y de sus hijos, en el marco de un discurso empático y de sensibilidad hacia la víctima.

Con respecto a los agresores, valoran la eficacia, la efectividad y la eficiencia de la Ley con relación a sus situaciones individuales, sin efectuar referencias explícitas al contenido de la Ley en sí misma. Por lo tanto, este discurso no hace una referencia directa a nuestros objetivos de investigación -como sucede con otros- sino que se presenta como una información latente bajo una capa de emocionalidad. En consecuencia, los victimarios han efectuado numerosas referencias a la justificación de la situación de violencia. Con carácter general, los agresores presentan distorsiones cognitivas, creencias irracionales y falta de asunción de responsabilidad (Boira y otros, 2010). Así mismo, han establecido férreos mecanismos de defensa. El grupo piensa que la ley es inadecuada, en tanto que infravalora y desprecia los hechos acaecidos, que ve como algo natural en una pareja. Por tanto, percibe la respuesta del Estado no acorde a lo que considera normal. Los agresores incluyen sus actos dentro del comportamiento habitual, de lo cotidiano, y entienden que son cosas que tienen que suceder en pareja: "y la he cogido de la mano y la he apretado demasiado, pumba, fuera. Ni le he pegado". Piensan que ante igual comportamiento de la mujer la justicia ofrece una distinta respuesta -discriminación-, esto es, observan respuestas diferentes de la justicia ante comportamientos similares en ambos miembros de la pareja: "Simplemente le respondí 
de la misma manera. Entonces lo que para mí se considera delito para ella no es nada, o sea, que a mí me condenan por eso sin haber hecho nada y a ella no le hacen nada". Con frecuencia alegan no ser responsables de sus actos y no los valoran como reprochables ni en el ámbito social, ni en el penal. Si bien, denotan como reprochables otras conductas que también presentan un rechazo social y penal:"hay miles de personas que han robado, pero están en la calle, ¿sabe por qué?”. Manifiestan una concepción errónea de quién es la víctima y quién el agresor, considerándose así mismos víctimas de su pareja y del Estado.

Por su parte, las víctimas manifiestan en su discurso poseer un nivel más alto de conocimiento del contenido de la Ley con referencias explícitas a la misma. De igual forma, la eficacia, la efectividad y la eficiencia de la Ley es valorada en función de las experiencias personales. Las víctimas describen las situaciones vividas de forma muy diferente a las de los victimarios, aunque indican que antes de romper la relación justificaban las situaciones de violencia de forma distorsionada (Walker, 1979; Escudero y otros, 2005) ${ }^{10}$. El discurso es realizado desde una perspectiva de género, obteniéndose continuas referencias al tema de la socialización desigual. La mediación es percibida como inviable para este tipo de supuestos. A su vez, en el discurso de las víctimas hay signos de reproche a la falta de sensibilidad y desatención asociada a la asistencia letrada y a la victimización secundaria.

\section{Discusión y conclusiones}

\subsection{Discusión}

La obtención de las categorías de análisis y su interpretación ha posibilitado dar respuesta a los objetivos de investigación -eficacia, efectividad y eficiencia- planteados. Cada uno de ellos incardina una o varias de las categorías estudiadas, no siendo todas susceptibles de inclusión.

La eficacia de la Ley ha sido ampliamente debatida en la categoría fundamentación de la Ley. La mayor parte de las percepciones englobadas aquí ponen

${ }^{10}$ Según diversos estudios, la situación de violencia se rompe definitivamente a raíz de un punto de inflexión o momento de crisis "tocar fondo". 
de relieve que no se ha producido una reducción de la violencia sobre la mujer pareja tras la promulgación de la normativa. Sin embargo, a pesar de ello opinan que la Ley es eficaz, puesto que los casos de muerte no son el único indicador objeto de valoración.

En cuanto a la efectividad, los agentes sociales consideran que la Ley es incapaz de poner en funcionamiento de forma adecuada todo el conjunto de recursos previstos en la norma, concretamente en lo que se refiere a recursos de corte social y preventivo. Todo ello, ha sido abarcado en las siguientes categorías temáticas: el desarrollo parcial de la Ley y los recursos previstos en la Ley.

Con relación a la eficiencia, se constata que existen costes ligados a la reducción de la violencia de género y que éstos son elevados. En la interposición de la denuncia, la mujer acude desinformada al sistema penal desconociendo las consecuencias de activar el procedimiento. Ello provoca que, tras la interposición de la misma, la mujer desista en el procedimiento e incluso conviva con el agresor sobre el que pesa una orden de alejamiento. A causa de lo anterior, en ocasiones, la mujer puede ser condenada como cómplice por un delito de quebrantamiento de condena (Montaner, 2007). Respecto a la intervención penal, los victimarios refieren que ésta no es igualitaria y que está siendo aplicada de forma injusta sobre los colectivos sociales más desfavorecidos. Esta afirmación se inserta en la categoría fundamentación errónea de la Ley y ha sido mencionada por un único grupo. Además, tanto los agresores como los operadores jurídicos, refieren que un mayor endurecimiento de las actuaciones de protección sobre la mujer -asociar la condena a la privación de la patria potestad- puede provocar efectos criminógenos. Por último, se alude a una sobrecarga de la gestión de las órdenes y penas de alejamiento en dos sentidos. En primer lugar, se debe otorgar de mayor discrecionalidad judicial a la orden y desligar su imposición a la condena. Ello queda reflejado en las categorías: el colapso del sistema penal y la orden de alejamiento. En segundo lugar, el hecho de abordar un problema social con una ley penal genera colapso y automatismo de los juzgados en la ejecución de las penas.

En conclusión, las hipótesis de partida han sido confirmadas parcialmente, a la vez que se ha recabado otra información relevante que amplía la investigación 


\subsection{Implicaciones}

A lo largo del presente estudio hemos tenido ocasión de conocer las implicaciones prácticas que conlleva la nueva legislación en materia de violencia de género. El carácter integral que la define condiciona en buena parte que su desarrollo tenga lugar en múltiples ámbitos de actuación, a la vez que exige la articulación y dotación numerosos recursos públicos. La falta de medios que posibiliten la consecución de sus fines preventivo generales, así como el desarrollo que se ha venido produciendo en su vertiente jurídico penal, se configuran como los principales problemas que ha tenido su puesta en marcha. La divergencia existente entre su fundamento teórico y su aplicación práctica hace que, con carácter general, la Ley sea percibida como un instrumento limitado en su propósito. En este sentido, hubiese sido oportuno efectuar una valoración político criminal ex ante dirigida a determinar los posibles impactos y costes asociados a su futura implementación. De haber sido así, se podría haber otorgado más relevancia al desarrollo de aspectos preventivos de la Ley, cuestión fundamental para que ésta despliegue sus efectos a largo plazo.

\section{Agradecimientos}

El presente estudio se integra en un proyecto de investigación denominado: "Los costes del enfoque punitivo para reducir la violencia sobre la mujer pareja" (SEJ-4161). Se trata de un proyecto de excelencia aprobado por la Junta de Andalucía en el año 2008. Las percepciones de los grupos sociales o usuarios a las que van dirigidas las políticas públicas existentes en una determinada sociedad se han configurado como una fuente de información rica para evaluar el funcionamiento de las mismas. En este sentido, quisiéramos agradecer la buena disposición manifestada por todas las personas que han participado en los grupos de discusión y subrayar que su colaboración ha sido imprescindible para llevar a cabo la investigación. En último lugar, hemos de precisar que el presente artículo muestra de manera sintetizada los principales resultados de un estudio más amplio que será publicado próximamente. 


\section{Referencias}

Boira, S., López, Y., Tomás, L. y Gaspar, A.R. (2010). Evaluación cualitativa de un programa de intervención psicológica con hombres violentos dentro de la pareja. Acciones e Investigaciones Sociales, 28, 135-156.

Conde, F. (2009). Análisis sociológico del sistema de discursos. Madrid: Centro de Investigaciones Sociológicas, colección Cuadernos Metodológicos, 43.

Escudero, A., Polo C., López M. y Aguilar L. (2005). La persuasión coercitiva, modelo explicativo del mantenimiento de las mujeres en una situación de violencia de género. Revista de la Asociación Española de Neuropsiquiatría, 25 (95), 85117.

Espinar, E. (2007). Las raíces socioculturales de la violencia de género. Escuela Abierta, 10, 23-48.

Flick, U. (2007). Introducción a la investigación cualitativa. Madrid: Ediciones Morata, S.L.

Gil, J. (1993). La metodología de investigación mediante grupos de discusión. Enseñanza \& Teaching: Revista interuniversitaria de didáctica, 10-11, 199-214.

Hallyday, M.A.K. (1982). El lenguaje como semiótica social. La interpretación socia del lenguaje y su significado. México: Fondo de Cultura Económica.

Ibáñez, R. (2000). Más allá de la sociología. El grupo de discusión: técnica y crítica Madrid: Siglo Veintiuno.

Íñiguez, L. (2006). Análisis del discurso: manual para las ciencias sociales. Barcelona: UOC.

Mena, A.M. y Méndez, J. M. (2009). La técnica de grupo de discusión en la investigación cualitativa: aportaciones para el análisis de los procesos de interacción. Revista Iberoamericana de Educación, 49 (3), 1-7.

Montaner, R. (2007. El quebrantamiento de penas o medidas de protección a las víctimas de violencia doméstica. InDret, 4, 1-26.

Osés, J. (2008). Evaluación legislativa y parlamento. Revista Debate, 5 (15), 58-69.

Ricoeur, P. (1995). Teoría de la Interpretación. Discurso y excedente de sentido. Madrid: Siglo XXI.

Roldán, E. (1998). Los grupos de discusión en la investigación en Trabajo Social y Servicios Sociales. Cuadernos de Trabajo Social, 11, 133-144. 
Ruiz, J. (2009). Análisis sociológico del discurso: métodos y lógicas. Forum Qualitative Socialforschung/Forum: Qualitative Social Research, 10 (2), 1-32. Disponible en: http://nbnresolving.de/um:nbn:de:0114-fqs0902263.

Ruiz, J. (2012). El grupo triangular: reflexiones metodológicas en torno a dos experiencias de investigación. Empiria: Revista de Metodología de Ciencias Sociales, 24, 141-162.

Walker, L.E. (1979). The battered women. New York: Harper and Row.

Lorea Arenas es licenciada en Criminología por la Universidad de Valencia. Posee un Máster en Criminalidad e Intervención Social en Menores y un Máster en Derecho Penal y Política Criminal por la Universidad de Málaga, así como estudios de posgrado en Métodos Avanzados de Estadística Aplicada e Investigación privada por la Universidad de Salamanca. En la actualidad es investigadora del Instituto andaluz interuniversitario de Criminología de la Universidad de Málaga donde ha participado en varios proyectos de investigación y donde desarrolla su tesis doctoral. Las principales temáticas que ha abordado en sus estudios han sido: criminología y nuevas tecnologías (TICs), violencia contra la mujer, métodos de investigación y estadística y seguridad pública.

Ana Isabel Cerezo es Profesora titular de Derecho penal y Subdirectora del Instituto andaluz interuniversitario de Criminología de la Universidad de Málaga. Becaria Fulbright en la School of Criminal Justice de Rutgers University (New Jersey-USA) en el 2001. Ha sido investigadora visitante en el Instituto de Criminología de Cambridge (UK) y profesora invitada en la Facultad de Derecho de Queensland University (Australia) y en la Universidad de Tsukuba (Japón). La mayor parte de sus publicaciones han seguido una línea de investigación con un marcado componente criminológico y victimológico, sin menospreciar sus trabajos en política criminal y derecho penal.

María José Benítez es Profesora sustituta interina de Derecho penal de la Universidad de Málaga. Ha sido Profesora investigadora en el Instituto andaluz interuniversitario de Criminología de la Universidad de Málaga desde 2005, miembro del Observatorio de la delincuencia (ODA) y técnico de apoyo en proyectos nacionales y autonómicos. Fue profesora en la Universidad de Castilla-La Mancha y coordinadora del Máster en Criminología de dicha universidad desde 2001 a 2003. Realizó su estancia posdoctoral en la Universidad de Manchester (UMIST) en 2004. Cuenta con publicaciones mayoritariamente criminológicas y ha colaborado con organismos públicos para la redacción de informes en esta materia. 